\section{Long-term follow up after third-party viral-specific cytotoxic lymphocytes for immunosuppression- and Epstein-Barr virus-associated lymphoproliferative disease}

Epstein-Barr virus (EBV)-associated lymphoproliferative disease is a serious complication of immunosuppression, particularly after transplantation. Treatment by reduction of immunosuppression risks graft loss after solid organ transplantation (SOT) and only $25-50 \%$ of patients respond. ${ }^{1,2}$ After hematopoietic stem cell transplantation (HSCT), it risks exacerbating graft-versus-host disease (GvHD) and by itself is usually ineffective, although it does represent a prerequisite for other therapies to be successful. ${ }^{3-6}$ Administration of rituximab can be efficacious as a monotherapy when used pre-emptively in response to rising EBV plasma titers or limited disease. ${ }^{6,7}$ In more advanced or refractory cases, it can be combined with chemotherapy. ${ }^{8-10}$ However, some patients fail to respond or are unsuitable for chemotherapy, and so are candidates for less toxic adoptive cellular therapy. ${ }^{11}$ Most experience has been gained using cytotoxic T-cell lines (CTL) stimulated by EBV transformed B-lymphoblastoid cell lines. ${ }^{11}$ Autologous- or donorderived cells are difficult to prepare in appropriate time frames and so difficult to use in the clinic. However, using third-party donors, cryopreserved cells can be banked for rapid issue. Our bank of EBV specific CTL was licensed in $2011^{12}$ and here we describe some successful outcomes for this difficult to treat group of patients.

When an enquiry is received (https://nhsnss.org/ services/blood-tissues-and-cells/clinical-services/cytotoxic-tcell-therapy/cytotoxic-t-cell-therapy-resources-for-clinicians/), details required include human leukocyte antigen (HLA) $-A,-B,-C,-D R B 1$ and -DQB1 types of patient and donor. No locus is preferentially matched, although CTL with matches at both HLA class I and II are preferred. If a patient has an anti-HLA antibody specific to a CTL, it is excluded. Cells sufficient for four doses of $1-2 \times 10^{6} / \mathrm{kg}$ body weight are shipped in nitrogen cryostorage for a course of four infusions given at weekly intervals. Cells are supplied on a non-profit making, cost-recovery basis (£12,500 per patient).

Data were collected with informed written consent from each patient and institutional approval from the Scottish National Blood Transfusion Service (SNBTS) Clinical Governance and Safety Committee. Complete responses (CR) were defined as disappearance of tumor masses or other disease manifestations, partial responses (PR) if tumors decreased in size or some disappeared while others remained, and no response (NR) when tumor masses remained the same or increased in size. Survival rates were calculated by non-parametric KaplanMeier analyses. Fisher exact tests were used to test whether ordinal characteristics were associated with response rates. $\chi^{2}$ trend tests were used for continuous variables. $P$-values are two-sided unless otherwise stated.

By the end of 2017, 132 HLA matching requests had been processed and 61 allocation reviews completed. There were a median of three class I matches (range, 0 6), two class II matches (range, 0-4) and 4.5 class I+II matches (range, 0-9). For CTL issued, there were a median of three class I matches (range, 1-6), two class II matches (range, 0-4), and five class I+II matches (range, 2-9) (Online Supplementary Figure S1). Cells were issued on 69 occasions: three patients were not infused (two died before treatment and one issue held as post-transplant reserve treatment). Two patients received a second course from different donors. Thus 64 patients received cells. Of the 59 recipients with over six months follow up, $34(58 \%)$ were male and $25(42 \%)$ female. Median age was 31 years (range, 1-82). Forty-eight patients had received transplants, 28 HSCT, and 20 SOT. All HSCT patients had received transplants from unrelated donors. SOT types were kidney $(n=10)$, liver $(n=3)$, heart $(n=2)$, bowel $(n=1)$, liver, small bowel and pancreas $(n=1)$, liver and kidney $(n=1)$, kidney and pancreas $(n=1)$, heart and kidney $(n=1)$. Of eleven non-transplant patients, five had congenital immunodeficiencies and four were receiving immunosuppressive drugs (2 for acute lymphoblastic leukemia, 1 each for Crohn disease and systemic lupus erythematosus). One patient had EBV-positive natural killer/T-cell lymphoma and one diffuse large B-cell lymphoma of the elderly.

Cells were infused in 14 UK cities and six other countries. Although CTL were available within days, median time from first contact to issue was 18 days (range, 1298). The most common reason for delay was obtaining funding. All patients had reduction of immunosuppression if possible. All patients had received prior rituximab and 18 had received additional chemotherapy. Thus CTL were mainly used for patients whose disease was refractory to reduction of immunosuppression and rituximab infusions, most often for patients not considered fit enough for any or additional cytotoxic chemotherapy.

Responses were observed in 35 of $59(59 \%)$ patients: $23(39 \%) \mathrm{CR}$ and $12(20 \%) \mathrm{PR}$. Of patients attaining complete remission, 19 of 23 were alive at the time of census, with four dying of other causes ( 2 infection and 2 relapsed primary disease) (Figure $1 \mathrm{~A}$ ). Of 12 patients achieving PR, four were alive at the time of census, all of whom received other forms of treatment after CTL infusions (chemotherapy, chemotherapy and excision, radiotherapy, chemotherapy including peripheral blood stem

Table 1. Response and survival rates by cause of immunosuppression.

\begin{tabular}{lccc} 
& HSCT & SOT & Other \\
Overall responses & $13 / 28(46 \%)$ & $15 / 20(75 \%)$ & $7 / 11(64 \%)$ \\
(CR/PR) & $(8 / 5)$ & $(10 / 5)$ & $(5 / 2)$ \\
\hline N. of deaths & $23 / 28(82 \%)$ & $8 / 20(40 \%)$ & $5 / 11(45 \%)$ \\
Median survival, years $(95 \% \mathrm{CI})$ & $0.1(0.05,0.15)$ & $3.87(0.00,8.66)$ & - \\
\hline Mean survival, years $(95 \% \mathrm{CI})$ & $1.0(0.40,1.67)$ & $2.79(1.90,3.67)$ & $3.16(0.86,4.85)$ \\
\hline
\end{tabular}

HSCT: hematopoietic stem cell transplantation; SOT: solid organ transplantation; CR: complete response; PR: partial response; N: number; CI: Confidence Interval. Although differences between response rates were not statistically significant (Fisher exact test, $P=0.13$ ), differences between survival times were significant (log rank Mantel-Cox test, $P=0.007$ ) 
cell transplant). No clinical responses were observed in 24 of $59(41 \%)$ patients, none of whom survived for more than three months. The condition of these patients had been very poor, with most dying before the schedule of the four infusions could be completed. Only nine received all four infusions, four received three, seven received two, and four patients received only a single infusion.

Outcomes from the non-transplant group were promising, with $64 \%$ response and $54 \%$ survival rates (Table 1 and Figure 1B). Responses were seen in three of the five with congenital deficiencies and CTL were used as a bridge to a successful HSCT. The response and survival rates of patients with post-transplant lymphoproliferative disease (PTLD) after solid organ transplantation were also good (75\% and $60 \%$, respectively); these are consistent with previous series ${ }^{13}$ and support the use of CTL in the treatment of rituximab resistant disease in this context.
However, the corresponding rates (46 and $18 \%$, respectively) from HSCT patients were worse than previously reported (Table 1 and Figure 1B). ${ }^{14}$ The lower response rate probably reflects the poor clinical status of many of the patients, with many dying before the infusions had been completed. The lower survival rate also probably reflects the propensity of such patients to die of causes other than PTLD. Uhlin et al. ${ }^{5}$ reported that the survival of patients with PTLD after HSCT is poor despite initial successful treatment, with 3-year survival rate $20 \%$ versus $62 \%$ without PTLD. Patients with bulky disease also fared poorly, indicating a high ratio of cytotoxic to target cells is important. It is thus of interest that excellent results have been reported using CTL as consolidation. ${ }^{15}$ There were no statistically significant differences in response rates when split by cause of immunosuppression (Fishers exact test, $P=0.13$ ) (Table 1). However, survival rates were significantly higher in patients who had not had a transplant or after undergo-

A

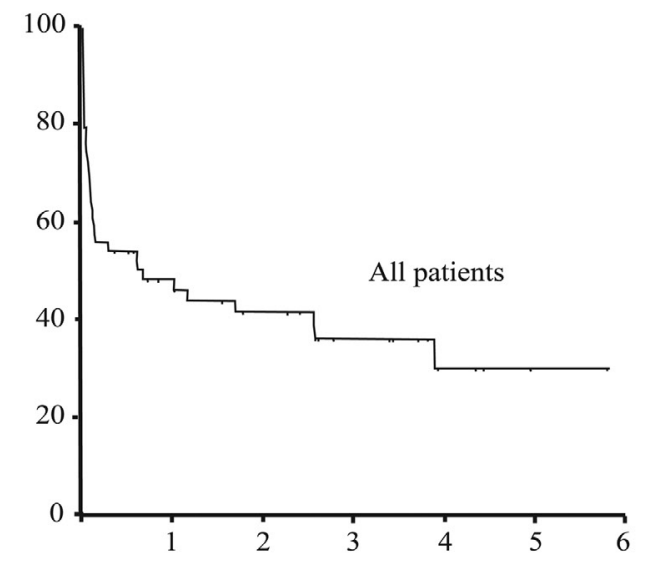

B

Survival after infusion $(\%)$

Survival after infusion $(\%)$

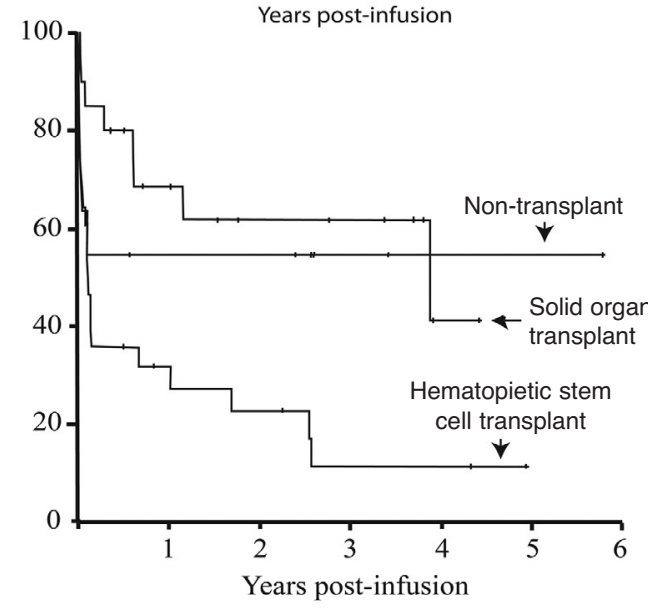

C

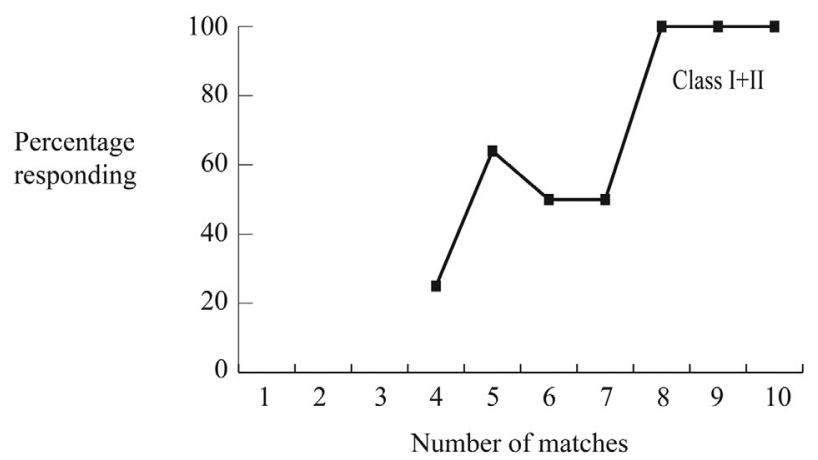

Figure 1. Kaplan-Meier survival curves and response rates by number of HLA matches. Kaplan-Meier survival curves plotted against time from end of infusions. (A) Data from all 59 patients. (B) Data split by non-transplant, solid organ transplant and hematopoietic stem cell transplant. No deaths after one year of infusion were ascribable directly to post-transplant lymphoproliferative disease (PTLD). (C) The proportion of patients exhibiting a clinical response, either complete response (CR) or partial response (PR), are plotted against number of HLA matches achieved. 
ing SOT versus HSCT (log rank, $P=0.007$ ) (Table 1).

Several clinical risk factors (sex, age, number of involved sites, extranodal disease) were investigated for prognostic significance, but no statistically significant differences were seen (Online Supplementary Table S1). However, a relatively high proportion (24 of $59,41 \%$ ), of patients had disease in their central nervous system (CNS), perhaps because of poor rituximab penetration. Overall, their response rate was $67 \%$ and survival at census was $50 \%$, better than those without CNS disease (54\% and $31 \%$, respectively) (Table 2). Outcomes were particularly good for the 13 with solitary lesions, 12 of whom $(92 \%)$ responded, and ten $(77 \%)$ were alive at the time of census (Fisher exact test, $P=0.012$ ). In contrast, five patients with multiple CNS lesions yielded only one CR (still alive at the time of census) and one PR. All three patients with diffuse CNS disease, two of whom also involved other organs, fared badly, with no responses and no survivors. An additional three with PTLD in other organs yielded two responses but only one was still alive at time of census.

Three patients had less conventional histology (Burkitt lymphoma, leiomyoma, natural killer cell); the outcomes from these patients was poor ( $\mathrm{PR}, \mathrm{PR}, \mathrm{NR}$, respectively) and all died.

Higher numbers of HLA matches were associated with better response rates (Figure $1 \mathrm{C}$ and Online Supplementary Table S2). Regarded as associations without prior mechanistic knowledge, these correlations failed to reach statistical significance (Fisher exact test, $P=0.4$ ). However, testing whether an increased number of matches results in the expected increase in response rates, using a one-tailed linear-by-linear association test, yields a $P$-value of 0.043 , with an effect of similar magnitude to previous reports. ${ }^{13}$ Doubrovina et al. reported elegant mechanistic data on the importance of appropriately HLA-matching third-party-derived CTL. ${ }^{14}$ Overall, it seems likely that there is an association, but its impact could be lessened because of other factors.

Cytotoxic T-cell lines were well tolerated with only two cases of mild cutaneous GvHD observed and a temporary worsening of neurological symptoms, thought to represent a tumor flare in a patient who responded.

In summary, we report outcomes from the use of thirdparty-derived, EBV-specific CTL, mainly used to treat patients with immunosuppression-associated lymphoproliferative disease who had failed conventional treatments or were too ill to tolerate chemotherapy. These data support the use of $\mathrm{T}$ cells in this difficult to treat group of patients, although results from their use after HSCT are a cause for concern and their use requires further optimization. Although the cost of these CTL compares favorably with other cellular therapy products, uptake from most European countries has been limited.

Sajida Kazi, ${ }^{1}$ Abhinav Mathur, ${ }^{2}$ Gwen Wilkie, ${ }^{3}$ Kirsty Cheal, ${ }^{4}$ Richard Battle, ${ }^{3}$ Neil McGowan, ${ }^{3}$ Neil Fraser, ${ }^{4}$ Emily Hargreaves, ${ }^{3}$ David Turner, ${ }^{3}$ John D.M. Campbell, ${ }^{3}$ Marc Turner ${ }^{3}$ and Mark A. Vickers ${ }^{1,2,4}$

${ }^{1}$ Department of Haematology, Aberdeen Roval Infirmary; ${ }^{2}$ University of Aberdeen; ${ }^{3}$ Scottish National Blood Transfusion Service, Edinburgh and ${ }^{4}$ Scottish National Blood Transfusion Service, Aberdeen, UK

Acknowledgments: we thank the clinicians, listed below, who provided care to these patients and obtained the clinical data: G Taylor, Caroline Duncan, Dominic Culligan, Aberdeen Royal Infirmary; I Singer, J Murphy, Monklands Hospital, Airdrie; Jane Hartley, Girish Gupte, Rosanna Gibbs, Sue Beath, Mordi
Table 2. Response rates in PTLD by clinical prognostic factors

\begin{tabular}{lcc} 
& Responder (\%) & Non-responder (\%) \\
Single CNS lesion & $12(92)$ & $1(8)$ \\
Multifocal CNS disease & $2(40)$ & $3(60)$ \\
\hline Diffuse CNS disease & $0(0)$ & $3(100)$ \\
CNS disease with & $2(40)$ & $3(60)$ \\
other organ involvement & & \\
\hline No CNS disease & $19(54)$ & $16(46)$ \\
\hline
\end{tabular}

The differences between the three main groups split by CNS disease (no CNS disease, single CNS lesion and multiple lesions (multifocal disease, diffuse CNS disease and CNS disease with other organ involvement) were statistically significant (Fisher exact test, $P=0.012$ ).

Muorah, Birmingham Children's Hospital; Bhuvan Kishore, Birmingham Heartlands Hospital; Rachel Protheroe, University Hospitals, Bristol; Ponni Sivaprakasam, Bristol Children's Hospital; Dr Lisa Sharkey, Addenbrookes Hospital, Cambridge; Neill Storrar, Angela Thomas, Susan Baird, Roval Hospital for Sick Children, Edinburgh; Angus Broom, Huw Roddie, Peter RE Johnson, Western General Hospital, Edinburgh; Michael Manson, Pam McKay, A Latif, I Novitzky-Basso, Pam McKay, N Heaney, Beatson West of Scotland Cancer Centre, Glasgow; F Pinto, Royal Hospital for Children, Glasgow; Katherine Hodgson, Ann Hunter, Leicester Royal Infirmary; A Patel, Rahuman Salim, Royal Liverpool University Hospital; S Agrawal, St Bartholomew's Hospital, London; Helen Jones/Caroline Booth, Judv Tavlor, Evalina London Children's Hospital; Winnie Ip, Juliana Silva, Persis Amrolia, Great Ormond Street Hospital, London; J Marsh, V Mehra, Victoria Potter, Kings College Hospital, London; A Karadimitris, Hammersmith Hospital, London; Kate Cwynarski, Mallika Sekhar, Royal Free Hospital, London; Piers Blombery, Elspeth Payne, Jonathan Lambert, Kirsty Thomson, Stephen Walsh,

Kate Cwynarski, Wendy Stewart, K Ediriwickrema, Stephen Mackinnon, University College Hospital London; Robert Wynn, Sarah Burns, Manchester Royal Infirmary; J Maddox, James Cook University Hospital, Middlesbrough; Mary Slatter, Andrew Gennery, Sophie Hambleton, Zohreh Nademi, Newcastle University \& Great North Children's Hospital, Royal Victoria Infirmary, Newcastle Upon Tyne; Michelle Lannon, Freeman Hospital, Newcastle Upon Tyne; Christopher P Fox, Nottingham University Hospitals; A Tsoulkani, N Russell, R Radia, Nottingham City Hospital; Loretta Ngu, Royal Devon and Exeter NHS Foundation Trust, Exeter; Norbert Stute, Harpreet Kaur, John Snowden, S Francis, Royal Hallamshire Hospital, Sheffield; Peter Flanagan, SS Brothers, Starship Children's Hospital, Auckland, New Zealand; D Turkiewicz, Skane University Hospital, Lund, Sweden; A Lankester, Leiden University Medical Centre, NL; Lars Vindelov, Rigshospialet, Copenhagen, Denmark;

Maher Gandhi, Princess Alexandra Hospital, Brisbane, Australia; Chris Fraser, Richard Mitchell, Lady Cliento Children's Hospital, Brisbane, Australia; Elisabeth Vandenberghe, St James Hosp, Dublin, Ireland; Heidi Segers, Marleen Renard, University Hospital, Leuven, Belgium. The authors would like to thank the Wellcome Trust for funding the initial set up of this bank, and the blood donors from New Zealand who provided cells.

Correspondence: MARK VICKERS.

m.a.vickers@abdn.ac.uk.

doi:10.3324/haematol.2018.207548

Information on authorship, contributions, and financial \& other disclosures was provided by the authors and is available with the online version of this article at WwW. haematologica.org.

\section{References}

1. Cesaro S, Pegoraro A, Tridello G, et al. A prospective study on modulation of immunosuppression for Epstein-Barr virus reactivation in 
pediatric patients who underwent unrelated hematopoietic stem-cell transplantation. Transplantation. 2010;89(12):1533-1540.

2. Weiner C, Weintraub L, Wistinghausen B, et al. Graft rejection in pediatric liver transplant patients with Epstein-Barr viremia and posttransplant lymphoproliferative disease. Pediatr Transplant. 2012; 16(5):458-464.

3. Styczynski J, Einsele H, Gil L, Ljungman P. Outcome of treatment of Epstein-Barr virus-related post-transplant lymphoproliferative disorder in hematopoietic stem cell recipients: a comprehensive review of reported cases. Transpl Infect Dis. 2009;11(5):383-392.

4. Parker A, Bowles K, Bradley JA, et al. Management of post-transplant lymphoproliferative disorder in adult solid organ transplant recipients - BCSH and BTS Guidelines. Br J Haematol. 2010;149(5):693705.

5. Uhlin M, Wikell H, Sundin M, et al. Risk factors for Epstein-Barr virus-related post-transplant lymphoproliferative disease after allogeneic hematopoietic stem cell transplantation. Haematologica. 2014;99(2):346-352.

6. Styczynski J, van der Velden W, Fox CP, et al. Management of Epstein-Barr Virus infections and post-transplant lymphoproliferative disorders in patients after allogeneic hematopoietic stem cell transplantation: Sixth European Conference on Infections in Leukemia (ECIL-6) guidelines. Haematologica. 2016;101(7):803-811.

7. Trappe RU, Dierickx D, Zimmermann $H$, et al. Response to Rituximab Induction Is a Predictive Marker in B-Cell Post-Transplant Lymphoproliferative Disorder and Allows Successful Stratification Into Rituximab or R-CHOP Consolidation in an International, Prospective, Multicenter Phase II Trial. J Clin Oncol. 2017;35(5):536543 .

8. Knight JS, Tsodikov A, Cibrik DM, Ross CW, Kaminski MS, Blayney DW. Lymphoma after solid organ transplantation: risk, response to therapy, and survival at a transplantation center. J Clin Oncol. 2009;
27(20):3354-3362.

9. Garcia-Cadenas I, Castillo N, Martino R, et al. Impact of Epstein Barr virus-related complications after high-risk allo-SCT in the era of preemptive rituximab. Bone Marrow Transplant. 2015;50(4):579-584.

10. Trappe R, Oertel S, Leblond V, et al. Sequential treatment with rituximab followed by CHOP chemotherapy in adult B-cell post-transplant lymphoproliferative disorder (PTLD): the prospective international multicentre phase 2 PTLD-1 trial. Lancet Oncol. 2012;13(2):196-206.

11. Bollard CM, Rooney CM, Heslop HE. T-cell therapy in the treatment of post-transplant lymphoproliferative disease. Nat Rev Clin Oncol. 2012;9(9):510-519.

12. Vickers MA, Wilkie GM, Robinson N, et al. Establishment and operation of a Good Manufacturing Practice-compliant allogeneic Epstein-Barr virus (EBV)-specific cytotoxic cell bank for the treatment of EBV-associated lymphoproliferative disease. Br J Haematol. 2014; 167(3):402-410.

13. Haque T, Wilkie GM, Jones MM, et al. Allogeneic cytotoxic T-cell therapy for EBV-positive posttransplantation lymphoproliferative disease: results of a phase 2 multicenter clinical trial. Blood. 2007; 110(4):1123-1131.

14. Doubrovina E, Oflaz-Sozmen B, Prockop SE, et al. Adoptive immunotherapy with unselected or EBV-specific T cells for biopsyproven EBV+ lymphomas after allogeneic hematopoietic cell transplantation. Blood. 2012;119(11):2644-2656.

15. Jiang X, Xu L, Zhang Y, et al. Rituximab-based treatments followed by adoptive cellular immunotherapy for biopsy-proven EBV-associated post-transplant lymphoproliferative disease in recipients of allogeneic hematopoietic stem cell transplantation. Oncoimmunology. 2016;5(5):e1139274. 